\title{
Cyclosporine A micellar delivery system for dry
}

\section{eyes}

\author{
This article was published in the following Dove Press journal: \\ International Journal of Nanomedicine \\ 21 June 2016 \\ Number of times this article has been viewed
}

\author{
Han Kang ${ }^{1,2}$ \\ Kwang-Ho Cha' \\ Wonkyung Cho' \\ Junsung Park' \\ Hee Jun Park' \\ Bo Kyung Sun ${ }^{1,2}$ \\ Sang-Min Hyun ${ }^{1,2}$ \\ Sung-Joo Hwang ${ }^{1,2}$ \\ 'Yonsei Institute of Pharmaceutical \\ Sciences, ${ }^{2}$ College of Pharmacy, Yonsei \\ University, Incheon, South Korea
}

Background: The objectives of this study were to develop stable cyclosporine A (CsA) ophthalmic micelle solutions for dry-eye syndrome and evaluate their physicochemical properties and therapeutic efficacy.

Materials and methods: CsA-micelle solutions (MS-CsA) were created by a simple method with Cremophor EL, ethanol, and phosphate buffer. We investigated the particle size, $\mathrm{pH}$, and osmolarity. In addition, long-term physical and chemical stability for MS-CsA was observed. To confirm the therapeutic efficacy, tear production in dry eye-induced rabbits was evaluated using the Schirmer tear test (STT). When compared to a commercial product, Restasis, MS-CsA demonstrated improvement in goblet-cell density and conjunctival epithelial morphology, as demonstrated in histological hematoxylin and eosin staining.

Results: MS-CsA had a smaller particle size (average diameter 14-18 nm) and a narrow size distribution. Physicochemical parameters, such as particle size, $\mathrm{pH}$, osmolarity, and remaining CsA concentration were all within the expected range of 60 days. STT scores significantly improved in MS-CsA treated groups $(P<0.05)$ in comparison to those of the Restasis-treated group. The number of goblet cells for rabbit conjunctivas after the administration of MS-CsA was $94.83 \pm 8.38$, a significantly higher result than the $65.17 \pm 11.51$ seen with Restasis. The conjunctival epithelial morphology of dry eye-induced rabbits thinned with loss of goblet cells. However, after 5 days of treatment with drug formulations, rabbit conjunctivas recovered epithelia and showed a relative increase in the number of goblet cells.

Conclusion: The results of this study indicate the potential use of a novel MS for the ophthalmic delivery of CsA in treating dry eyes.

Keywords: cyclosporine A, Cremophor EL, micelle solution, dry eyes, Restasis

\section{Introduction}

Cyclosporine A (CsA) is neutral and lipophilic. It is made of a cyclic undecapeptide of amino acids that contain four intramolecular hydrogen bonds. It is isolated from the Tolypocladium inflatum Gams and other Fungi imperfecti. ${ }^{1-4} \mathrm{CsA}$ is a powerful immunosuppressive drug, now routinely utilized to prevent rejection of transplanted organs. It acts by selectively inhibiting T cells, and thus causes suppression of the cell-mediated immune response. ${ }^{1,5}$ Because of its hydrophobicity with poor water solubility $(0.012 \mathrm{mg} / \mathrm{mL}$ at $25^{\circ} \mathrm{C}$ ), CsA must be solubilized in nonaqueous media that include surfactants, such as Cremophor EL, as in the case of Sandimmune ${ }^{\circledR}$ (Novartis International AG, Basel, Switzerland). ${ }^{6-8}$ As an immunosuppressant, CsA at a low concentration has been shown to be useful for patients with inflammatory ocular surface disorders, including dryeye syndrome. Dry-eye syndrome is a disorder of the tear film, occurring as a result of tear deficiency or excessive evaporation. Dry-eye syndrome causes damage to the interpalpebral ocular surface, and large numbers of patients suffer from symptoms of
Correspondence: Sung-Joo Hwang College of Pharmacy, Yonsei University, 85 Songdogwahak-ro, Yeonsu-gu, Incheon 21983, South Korea

Tel $+823274945 I 8$

Fax +82327494105

Email sjhI I@yonsei.ac.kr 
discomfort. $^{9-11}$ Topical administration is the main route for the treatment of dry-eye syndrome, and the targeted organs are the cornea, conjunctiva, or lachrymal gland. ${ }^{1}$

A commercial CsA product for ophthalmic formulation is $\operatorname{Restasis}^{\circledR}$ (0.5 mg/mL CsA; Allergan Inc, Irvine, CA, USA). This product is an oil-in-water emulsion eyedrop that contains a mixture of castor oil and Tween 80 . However, the most common side effect of castor oil is ocular burning or irritation following the chronic use of eyedrops. In addition, adverse effects, such as itching, redness, visual disturbance, and eye pain, are problems that prevent patients from using these eyedrops. Poor ocular tolerance, low bioavailability, and instability are other major drawbacks reported with Restasis. ${ }^{12,13}$ These difficulties may be overcome through various drug formulations. Many studies have attempted to improve the availability and tolerance of topically applied CsA through the development of such forms as cosolvency, prodrugs, emulsions, and colloidal systems. Nevertheless, almost none of the delivery systems has been fully satisfactory. ${ }^{1,7}$ Ikervis $^{\circledR}$ (1 mg/mL CsA; Santen Pharmaceutical Co Ltd, Osaka, Japan), which has recently been introduced to EU markets, is a CsA formulation that can help alleviate dry-eye syndrome. However, its milky white emulsion poses some challenges, especially patient compliance.

There has recently been a great deal of attention focused on micelle formulations. Micelles have been useful in the solubilization of water-insoluble drugs, such as CsA. Also, CsA in a micellar structure is stabilized in a biological environment. ${ }^{14}$ For instance, nanoscale micelles as drug carriers are promising for being utilized as topical ophthalmic administration, because of their excellent biocompatibility and ocular tolerance. ${ }^{7}$ Therefore, a micelle formulation is effective in delivering CsA into corneal layers and ocular tissues, allowing successful local CsA action. However, thus far there have been few studies on CsA-micelle formulations used to treat dry-eye syndrome. Kuwano et al solubilized CsA in isotonic and neutral aqueous solution using micelles of the nonionic surfactant - polyoxyl 40 stearate. ${ }^{15}$ Although this work was promising, the ocular tolerance of polyoxyl 40 stearate is not yet known and has not been evaluated. Recently, there have been studies on ocular delivery of transparent nanomicellar CsA, but these have been hampered by difficulties in the manufacturing process, which requires evaporation and film rehydration. In addition, these studies were not able to confirm therapeutic efficacy, measured by recovery of goblet cells and increase in aqueous tear production. ${ }^{16,17}$ Therefore, further research is essential to the development of the preferred micellar formulation.
Nonionic surfactants are used mainly in ophthalmic delivery systems because of their excellent compatibility and stability. Furthermore, with milder impact on cell membranes in comparison to that posed by more toxic cationic or anionic surfactants, nonionic surfactants are less irritating. ${ }^{18}$ Among the nonionic surfactants, Tween 80 and Cremophor EL are generally nonirritating or are known to cause only mild redness in the eye of the rabbit. Mild redness has been shown to disappear within a few hours (the rabbit eye is more susceptible to irritant substances than the human eye). ${ }^{19,20}$ However, Cremophor EL enhances the permeation of CsA through human corneas. It was found that the presence of Cremophor EL led to more drug bioavailability compared to Tween $80 .^{20,21}$ Therefore, the aim of this study was to evaluate CsA-micelle solutions (MS-CsA) containing Cremophor EL prepared using a simple method. The efficiency of MS-CsA in producing tears was tested in rabbit (dry-eye induced) eyes by analyzing the changes in the Schirmer tear test (STT). Goblet cell-density determination and epithelium-morphology evaluation of rabbit conjunctivas were also carried out to investigate the strength of the newly proposed MSs.

\section{Materials and methods Materials}

CsA was obtained from Concord Drugs Ltd (Hyderabad, India). Polyoxyl 35 castor oil (Cremophor EL) was generously gifted by BASF Co Ltd (Ludwigshafen, Germany). Glycerin (purity 99\%), ethanol (purity 99.9\%), sodium phosphate dibasic (anhydrous), sodium phosphate monobasic (anhydrous), and high-performance liquid chromatography (HPLC)-grade acetonitrile were purchased from Samchun Pure Chemical Co Ltd (Pyeongtaek, South Korea). Restasis emulsion was purchased from a local market. Atropine sulfate (AS) and hematoxylin and eosin (H\&E) were purchased from Sigma-Aldrich (St Louis, MO, USA). Purified water (Milli-Q; EMD Millipore, Billerica, MA, USA) was used through the study. All other chemicals used were of analytical grade.

\section{Preparation of CsA-micelle solutions}

The MS-CsA were prepared using very simple method. Formulations of MS-CsA containing 0.01-5 (weight $\%$ ) Cremophor EL were used in this study. To explain briefly, the CsA was dissolved in surfactant with ethanol $(0,0.5$, and 1 weight $\%$ ). Sodium phosphate dibasic, sodium phosphate monobasic, and glycerin (2.2 weight $\%$ ) were dissolved in the aqueous phase. We adjusted the volume of the water phase to be $100 \mathrm{~mL}$ when both solutions were mixed. Both solutions 
were stirred with a magnetic stirrer separately until a homogeneous state was obtained. The homogeneous mixture was selected by visual observation. After the homogeneous solution was obtained, two solutions were mixed. If the resulting formulation was not miscible, stirring with a magnetic stirrer was given an additional run. The $\mathrm{pH}$ was adjusted to 7.4, which was suitable for topical application to ocular tissue by phosphate ratio (Sørensen's phosphate buffer, $\mathrm{pH}$ 7.4). The micelle formulation was then filtered through a polytetrafluoroethylene-membrane filter (GE Healthcare Life Sciences, Little Chalfont, UK) with a pore size of $0.2 \mu \mathrm{m}$. All processes were carried out at room temperature.

\section{Characterization of MS-CsA}

\section{Analysis of particle size}

The average diameter and size distribution of MS-CsA and Restasis were measured by dynamic light scattering using an electrophoretic light-scattering spectrophotometer (ELSZ; Otsuka Electronics, Hirakata, Japan). The laser source was the semiconductor diode, and the measurement angle was $165^{\circ}$. We did not dilute the MS-CsA or Restasis, which were both measured by adjusting the intensity. Measurements were carried out at room temperature. Particle sizes are expressed as volume mean diameter in nanometers.

\section{Determination of $\mathrm{pH}$ and osmolarity}

The $\mathrm{pH}$ was examined by a $\mathrm{pH}$ meter (S20-KS; Mettler Toledo, Columbus, OH, USA). Experiments were executed at room temperature. The osmolarity of MS-CsA and Restasis was analyzed by an osmometer (K-7000; Knauer, Berlin, Germany). The interior temperature of the osmometer was controlled at $45^{\circ} \mathrm{C}$. A $400 \mathrm{mOsm} / \mathrm{kg}(12,687 \mathrm{~g} \mathrm{NaCl} / \mathrm{kg})$ $2 \mathrm{~mL}$ Eichlösung ampoule was used for calibration of the osmometer.

\section{Analysis of CsA content}

CsA content was determined by HPLC. HPLC analysis of CsA was performed using a method similar to that utilized in our previous study. ${ }^{12}$ The system was an HPLC set (Agilent Technologies, Santa Clara, CA, USA), which consisted of a pump (model 1260 quaternary pump VL), an autosampler (model 1260 ALS), and an ultraviolet detector (model 1260 VWD VL). A C18 reverse-phase column (Eclipse XDB $\mathrm{C} 18,5 \mu \mathrm{m}, 4.6 \times 250 \mathrm{~mm}$; Agilent) was used at $70^{\circ} \mathrm{C}$. The mobile phase consisted of acetonitrile:water (90:10), and was pumped at a flow rate of $1 \mathrm{~mL} / \mathrm{min}$. The eluent was monitored at $210 \mathrm{~nm}$ following injections of $20 \mu \mathrm{L}$ standard CsA solutions in ethanol and MS samples. At specific time intervals, each sample was immediately filtered through the $0.45 \mu \mathrm{m}$ polytetrafluoroethylene-membrane filter prior to analysis.

\section{Stability studies of MS-CsA}

For a stability test of the MS-CsA, MSs were stored at $4^{\circ} \mathrm{C}$ and also at room temperature. The samples were protected from light. The parameters of particle size, $\mathrm{pH}$, and osmolarity were chosen to study the stability over a period of 2 months. Particle sizes were measured using the ELSZ particle-size analyzer. Also, suitable aliquots of the samples were collected at given times $(0,4,7,10,14,21,30$, and 60 days), and the concentration of the remaining CsA was analyzed by HPLC. The degree of aggregation and phase separation were assessed visually at specific time intervals. All other visible changes were recorded.

\section{In vivo study of MS-CsA in dry-eye syndrome-induced rabbit model}

\section{Animal treatments}

Unanesthetized, fully awake male New Zealand albino rabbits (Samtako Bio Korea Inc, Osan, South Korea) weighing $1.5-1.8 \mathrm{~kg}$ were used throughout. The animals were housed singly in standard cages at $19^{\circ} \mathrm{C} \pm 1^{\circ} \mathrm{C}$ and $50 \% \pm 5 \%$ relative humidity, with proper light conditions (12-hour light-dark cycle). They were fed a standard diet and purified water. They were allowed to move their heads freely, and their eye movements were not restricted. ${ }^{22}$ All the experiments received the approval from the Committee on Care and Use of Laboratory Animals at Yonsei University, Incheon, South Korea and were carried out according to the Guidelines.

The animals were divided into a control and three different drug-treated groups, each group consisting of eight units. The rabbits were preliminarily given the STT. To induce dry eyes, $1 \%$ AS (Sigma-Aldrich) solution was instilled into both eyes of the rabbits. At 9 am, 2 pm, and 7 pm, three groups received $50 \mu \mathrm{L}$ AS solution in the lower conjunctival sac of both eyes. Five minutes after each administration of AS solution, each group received $50 \mu \mathrm{L}$ normal saline (NS) in one eye. The other eye received drug treatment, which was MS-CsA (O4 or O7) or commercial CsA emulsion (Restasis). The fourth (control) group received $50 \mu \mathrm{L}$ of AS solution or NS in each eye, as the only treatment, at $9 \mathrm{am}, 2 \mathrm{pm}$, and $7 \mathrm{pm}$. All treatments were continued for 5 days.

\section{Measurement of tear production \\ (Schirmer tear test)}

The ability to measure tear production is important for the diagnosis of many types of lachrymal dysfunction, including 
dry eyes. The STT measured the aqueous portion of tear secretion in response to both conjunctival stimulations. ${ }^{23}$ Tear production was measured with the STT using sterile test strips (ColorBarTM; Eagle Vision Inc, Memphis, TN, USA), which were made of $5 \times 35 \mathrm{~mm}$ standardized filter paper strips with a blue color bar that moves with a tear front. The test was executed on both eyes (unanesthetized) of all animals, by maintaining test strip in the external lower conjunctival sac for 2 minutes. The wetted length in millimeters of the strip was taken as the test score. In our study, after causing dry eye in both eyes, we confirmed the difference between the administration of the drug (CsA) formulations (MS-CsA and Restasis) and NS. The tear-difference value was calculated from equation 1:

$$
\text { Tear-difference value }(\mathrm{mm})=\mathrm{S}_{\mathrm{t}}-\mathrm{S}_{\mathrm{c}}
$$

where $\mathrm{S}_{\mathrm{t}}$ is the STT scores of drug formulation-treated eye, and $\mathrm{S}_{\mathrm{c}}$ the STT scores of NS-treated eyes. This equation was corrected for variation owing to individual difference. The STT was performed at 9 pm on day 1 , day 3 , and day 5 .

\section{Histologic analysis}

Conjunctival biopsy specimens measuring approximately $5 \times 5 \mathrm{~mm}$ were obtained from the same regions in the bulbar conjunctiva by surgeons following standard procedures, in control eyes and eyes of rabbits treated with the drug formulations. Removed eye tissues from rabbits were fixed in formalin solution and embedded in paraffin. Eye tissues were then cut into $4 \mu \mathrm{m}$-thick sections at room temperature. After deparaffinization, $4 \mu \mathrm{m}$ sections were processed and mounted on gelatin-coated slides. These were then stained with H\&E. ${ }^{24,25}$

\section{Goblet cell-morphology determination}

Conjunctival biopsies were performed to evaluate conjunctival goblet-cell density and epithelial morphology. Morphology was assessed with a bright-field microscope (BX-51; Olympus, Tokyo, Japan) with magnification of 40×. Conjunctival epithelial morphology was evaluated, and the numbers of conjunctival epithelial goblet cells were counted along the length of three separate tissues by two independent masked observers. ${ }^{24}$

To study the direct effects of AS and NS on the ocular surface, $50 \mu \mathrm{L} 1 \%$ AS solution and $50 \mu \mathrm{L} 0.9 \%$ NS were applied to the ocular surface for 5 days. To evaluate the curative value, $50 \mu \mathrm{L}$ drug formulations (MS-CsA and Restasis) were applied, according to the method described previously. Conjunctival biopsies were then performed to evaluate conjunctival goblet-cell density and epithelial morphology.

\section{Statistical analysis}

Statistical analysis was carried out using Student's $t$-test for tear production and goblet-cell counting. One-way analysis of variance (ANOVA) tests were performed to compare the means of more than two groups. The level of significance was set at $P<0.05$. All results are expressed as mean \pm standard deviation.

\section{Results and discussion Determination of optimal MS-CsA}

In this study, it was necessary to find optimal formulations for transparent MS. MS-CsA of various formulations was considered, and CsA $0.05 \%$ and $0.1 \%$ were deemed the most appropriate concentrations for ophthalmic formulations, because no additional benefits were observed with higher CsA concentrations. In addition, CsA $0.05 \%$ produced the most consistent improvements in patient symptoms, such as ocular dryness. ${ }^{26}$

Ethanol was selected as the cosolvent in the US Food and Drug Administration's acceptable range for the ophthalmic route. All formulations contained 2.2\% glycerin. We conducted a study on compositions containing various concentrations of ethanol and Cremophor EL.

The results obtained with various micelle formulations are summarized in Table 1. First, formulations of MS-CsA containing 0.01-1 (weight\%) Cremophor EL without ethanol were not manufactured $(\mathrm{Z} 1-\mathrm{Z} 4)$, because in the process of dissolving the CsA in a surfactant, CsA did not get wet enough in these compositions. Furthermore, the formulations of MS-CsA containing 0.01-0.5 (weight\%) Cremophor EL with ethanol $(0.5 \%$ and $1 \%)$ were not transparent $(\mathrm{F} 1-\mathrm{F} 3$, O1-O3). These formulations had large mean diameters of 160-360 nm, with no homogeneous distribution. Once a clear MS was created, the composition did not seem to have a perceivable effect on particle size. Therefore, further experiments thereafter for MS-CsA were all performed with MSs with a size less than $20 \mathrm{~nm}$.

For the stability test, Z5-Z7, F5-F7, and O5-O7 were selected. All of these samples were very stable, as seen from the stability-test results, yet of these samples, 07 was selected to be compared to Restasis. Aqueous tear production and goblet-cell morphology were the comparison criteria used to compare $\mathrm{O} 7$ with Restasis. van der Bijl et al showed that Cremophor EL improved the permeability of CsA through the 
Table I Particle size and transparency of different micelle solutions

\begin{tabular}{|c|c|c|c|c|c|}
\hline \multirow[t]{2}{*}{ Formulation } & \multicolumn{3}{|c|}{ Composition (weight\%) } & \multicolumn{2}{|l|}{ Particle size (nm) } \\
\hline & Cremophor EL & Ethanol & Glycerin & Mean diameter & Transparency \\
\hline \multicolumn{6}{|c|}{ Ethanol 0 system } \\
\hline $\mathrm{ZI}$ & 0.01 & 0 & 2.2 & - & - \\
\hline $\mathrm{Z2}$ & 0.05 & 0 & 2.2 & - & - \\
\hline Z3 & 0.5 & 0 & 2.2 & - & - \\
\hline $\mathrm{Z} 4$ & 1 & 0 & 2.2 & - & - \\
\hline Z5 & 3 & 0 & 2.2 & $17.50 \pm 0.26$ & $\checkmark$ \\
\hline Z6 & 4 & 0 & 2.2 & $17.73 \pm 0.15$ & $\checkmark$ \\
\hline $\mathrm{Z7}$ & 5 & 0 & 2.2 & $|7.27 \pm 0.3|$ & $\checkmark$ \\
\hline \multicolumn{6}{|c|}{ Ethanol $0.5 \%$ system } \\
\hline $\mathrm{FI}$ & 0.01 & 0.5 & 2.2 & $359.43 \pm 6.1$ & $x$ \\
\hline F2 & 0.05 & 0.5 & 2.2 & $192.13 \pm 3.76$ & $\times$ \\
\hline F3 & 0.5 & 0.5 & 2.2 & $213.93 \pm 6.97$ & $\times$ \\
\hline F4 & 1 & 0.5 & 2.2 & $|4.73 \pm 0.2|$ & $\checkmark$ \\
\hline F5 & 3 & 0.5 & 2.2 & $17.3 \pm 0.1$ & $\checkmark$ \\
\hline F6 & 4 & 0.5 & 2.2 & $17.53 \pm 0.38$ & $\checkmark$ \\
\hline F7 & 5 & 0.5 & 2.2 & $17.33 \pm 0.29$ & $\checkmark$ \\
\hline \multicolumn{6}{|c|}{ Ethanol I\% system } \\
\hline OI & 0.01 & I & 2.2 & $199.77 \pm 7.23$ & $\times$ \\
\hline $\mathrm{O} 2$ & 0.05 & I & 2.2 & $168.1 \pm 2.4$ & $\times$ \\
\hline $\mathrm{O} 3$ & 0.5 & I & 2.2 & $245.17 \pm 4.31$ & $\times$ \\
\hline O4 & 1 & I & 2.2 & $14.53 \pm 0.4$ & $\checkmark$ \\
\hline O5 & 3 & I & 2.2 & $17.37 \pm 0.25$ & $\checkmark$ \\
\hline O6 & 4 & I & 2.2 & $17.53 \pm 0.06$ & $\checkmark$ \\
\hline O7 & 5 & I & 2.2 & $17.2 \pm 0.3$ & $\checkmark$ \\
\hline
\end{tabular}

Notes: ZI-Z4 did not form micelle solutions in this study. Transparency was assessed visually. Data expressed as mean \pm standard deviation ( $=3$ ). " $\checkmark$ ” Transparent micelle solution; " $\times$ ” non-transparent solution; “-” not formed solution.

rabbit cornea, and so for this reason $\mathrm{O} 7$ with $5 \%$ Cremophor EL was selected. ${ }^{27}$

Unlike the commercial product, which is an opaque emulsion, the MS-CsA we prepared was transparent. This difference is expected to improve the therapeutic effect and reduce the irritation. MSs that do not contain oil, which is responsible for causing irritation in eyedrops, are expected to decrease irritation. In fact, we have registered a patent (Korean patent 10-1211902) for a nonirritating eyedrop that is the focus of this study. In addition, the micellar formulations proposed in this study need just a simple experimental setup with low cost. This novel nanoscale MS-CsA formulation will enhance the ocular delivery potential of CsA.

\section{Particle size and distribution of MS-CsA}

It is well known that the critical micelle concentration (CMC) of a given surfactant aqueous solution increases with the addition of a cosolvent. Although ethanol acts as a cosolvent at higher concentrations, it acts as a cosurfactant at lower concentrations and thus rather reduces the CMC. ${ }^{28}$ In addition, we also took into account the existence of other molecules and the peptide environment that could affect the CMC. It was determined that interference from other molecules may also raise the CMC value of Cremophor EL in plasma. ${ }^{29}$ For this reason, even when Cremophor EL has a higher concentration than the $\mathrm{CMC}$, micelles are not necessarily formed. Therefore, considering surfactant interaction with peptides, drugs, and various excipients is very important. We empirically determined the range in which micelles are formed. In our study, a transparent MS was not formed at 0.5\% Cremophor EL formulations of high concentration compared to the CMC of Cremophor EL (0.02 wt\%). ${ }^{18} \mathrm{As}$ a result, we conducted an experiment with formulations containing more than $1 \%$ Cremophor EL.

As shown in Table 1, formulations containing 1\% or more Cremophor EL were clear MSs, except for Z4. (As mentioned earlier in the manuscript, Z4 was not manufactured.) The average diameter of MS-CsA ranged from $14 \mathrm{~nm}$ to $18 \mathrm{~nm}$. In general, it is known that the pharmaceutical micelle has a size range of 10-80 nm (of note, particle sizes of micelles have a range of 5-100 nm). Micelles with a size range of 10-20 nm particularly enhance the ability to penetrate various tissues, and this size range also allows them to remain stable in vivo for a sufficiently long time. This size range also reduces biological side effects, and within this size range, the carrier components are thought 

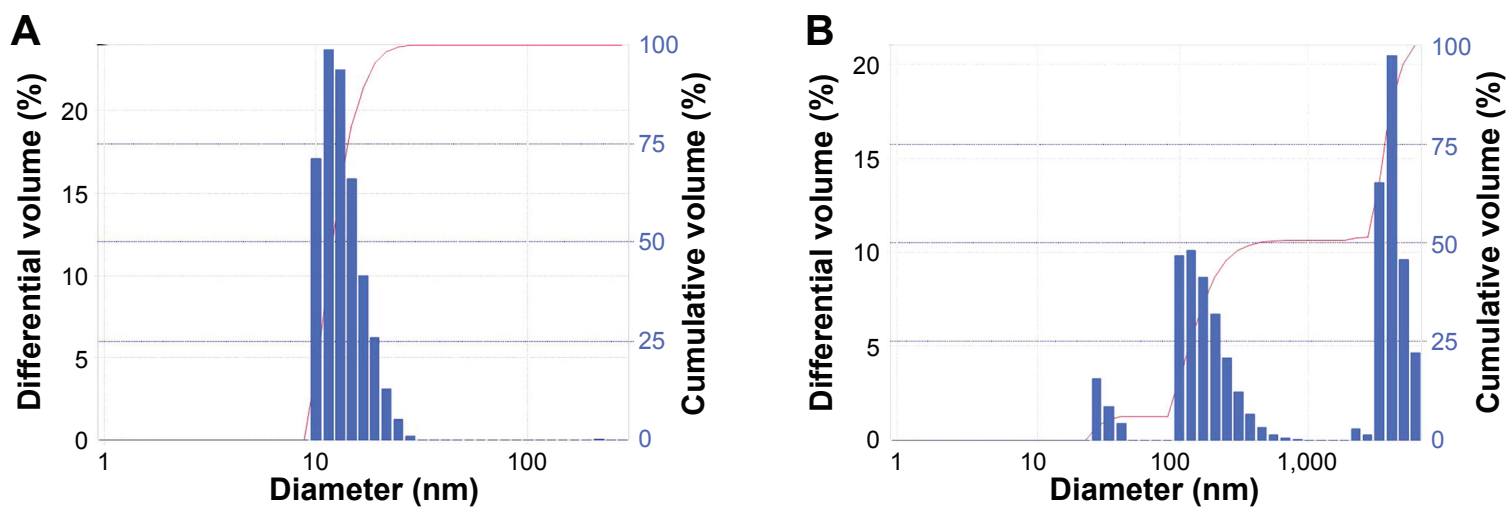

Figure I Size distribution (by volume) of CsA eyedrops obtained by DLS for (A) O7 MS and (B) Restasis.

Note: Bar refers to the differential volume (\%), and line graph refers to the cumulative volume (\%).

Abbreviations: CsA, cyclosporine A; DLS, dynamic light scattering; MS, micelle solution.

to leave the body easily when the therapeutic function is completed. .,30 $^{-30}$

Unlike opaque Restasis, MS-CsA is supposed to be more homogeneously distributed. This can be further verified by Figure 1, which represents the volume-distribution curves of the formulation $\mathrm{O} 7$ and Restasis. Distribution results indicate that Restasis achieved more peaks than MSCsA. These results proved that MS-CsA was smaller and more homogeneous compared with the commercial CsA product.

\section{$\mathrm{pH}$ and osmolarity studies}

Ophthalmic medication and ocular disease may alter corneal $\mathrm{pH}$, affecting functions of eye tissue. ${ }^{31}$ Shifts in ocular surface $\mathrm{pH}$ also could have therapeutic and diagnostic implications. $\mathrm{pH}$ changes could affect drug effectiveness and clinical signs in the disease process. ${ }^{32}$ Therefore, $\mathrm{pH}$ analysis of the prepared eyedrops was essential. The $\mathrm{pH}$ and buffering capacity of an ophthalmic preparation are important, since the stability of commonly used ophthalmic drugs is generally controlled by the $\mathrm{pH}$ of their environment. In addition to stability effects, $\mathrm{pH}$ adjustment can influence comfort, safety, and activity of the ocular products. ${ }^{33}$ Normal tears have a $\mathrm{pH}$ of about 7.4, and possess some buffer capacity (US Pharmacopoeia; USP 29-NF 24, Ophthalmic preparations). Normally, the buffering action of the tear is capable of neutralizing topically applied eyedrops, and is thus able to prevent marked discomfort. To maximize comfort, an ophthalmic preparation should have the same $\mathrm{pH}$ as the lachrymal fluid. Therefore, we produced MS-CsA of $\mathrm{pH} 7.4$ with maximum buffer capacity within the allowable range of osmolarity.

Tear osmolarity correlated significantly with dry-eye syndrome, as progressively elevated tear osmolarity related to the worsening of disease severity. Although it is important to keep in mind that tear osmolarity cannot be used as the sole indicator of dry-eye syndrome, tear osmolarity as a biomarker for dry-eye disease severity is a matter to be considered. ${ }^{34}$ Therefore, the osmolarity of drug formulations that are used for eyedrops should be both confirmed and controlled. Lachrymal fluid is almost isotonic to $0.9 \%$ sodium chloride solution, which has osmolarity of approximately $300 \mathrm{mOsm}$. However, the eye can tolerate a range of osmolarity between $0.6 \%$ and $2 \% \mathrm{NaCl}$ solution, and so we adjusted osmolarity within this range.

The $\mathrm{pH}$ and osmolarity of the MS-CsA were in the expected range as follows. The $\mathrm{pH}$ range of prepared MS-CsA was 7.41-7.47, and the osmolarity range of prepared MS-CsA was 298.3-317.4. These results can be regarded as the range to minimize irritation in the application of the eyedrops. Eye irritation is accompanied by eye blinks and more tear secretion, as a defense mechanism for the recovery of normal conditions. When confronted with these discomforts, the defense mechanism may be more rapid loss of the drug with a reduction in therapeutic response. ${ }^{33}$ Therefore, to prevent these problems, appropriate ranges of $\mathrm{pH}$ and osmolarity for our MS-CsA were carefully determined.

\section{Stability of MS-CsA}

Stability is a critical factor that must be considered during formulation design and development. Physical and chemical stability is one of the major barriers that limit the widespread use of CsA as pharmaceutical eyedrops. As a dry powder, CsA is very stable for at least 2 years under dark and refrigerated $\left(2^{\circ} \mathrm{C}-8^{\circ} \mathrm{C}\right)$ conditions and over 7 months at $40^{\circ} \mathrm{C}$, $75 \%$ relative humidity. ${ }^{35}$ In contrast, it is difficult to prepare aqueous ophthalmic CsA, because of both its hydrophobicity $\left(\log _{P}=2.92\right)^{36}$ and its extremely low aqueous solubility $(6.6 \mu \mathrm{g} / \mathrm{mL}){ }^{37}$ Therefore, in most other studies, CsA was 
dissolved and administered in vegetable oils, such as the castor oil contained in Restasis. However, these vegetable oils may present problems of stability, such as rancidity. ${ }^{1}$ These difficulties may be overcome through formulations aimed at improving CsA water solubility (eg, micelles) and ensuring stability. Therefore, stabilization of CsA in aqueous conditions was an important factor in the development of CsA ophthalmic formulations. The results showed that the micelle formulations of our study contribute to the stabilization of the CsA to a great degree.

The stability of MSs was evaluated using MS-CsA of less than $20 \mathrm{~nm}$. Z5-Z7, F5-F7, and O5-O7 were used for this study. The initial size range of selected MS-CsA was 17.2$17.73 \mathrm{~nm}$. The particle-size range of these samples was maintained at $18.53-19.63 \mathrm{~nm}$ after 60 days of storage at room temperature, and was also maintained at 18.03-19.27 nm after 60 days of storage at $4^{\circ} \mathrm{C}$. The results of particle-size analysis during the 60 days of storage at room temperature and at $4^{\circ} \mathrm{C}$ are presented in Table 2 . It was interesting to note that MS-CsA did not show noticeable changes in particle size during the 60 days. Also, visible changes, such as aggregation and phase separation, were not observed, indicating concordance with the size results. In other words, these MS-CsA were physically stable when stored at both temperatures.

Table 3 shows the stability of MS-CsA with respect to changes in $\mathrm{pH}$ and osmolarity during 60 days of storage at room temperature and at $4^{\circ} \mathrm{C}$. The increasing ratio of $\mathrm{pH}$ at room temperature was $0.992-1$ and was $0.987-0.999$ at $4^{\circ} \mathrm{C}$.
Furthermore, the increasing ratio of osmolarity at room temperature was $0.947-1.02$ and was $0.959-1.012$ at $4^{\circ} \mathrm{C}$. Stability results of $\mathrm{pH}$ and osmolarity showed no remarkable changes during the 60 days.

Figure 2 shows the chemical stability during 60 days of storage at room temperature and at $4{ }^{\circ} \mathrm{C}$. The initial concentrations of remaining CsA were 96.11\% $\pm 2.26 \%$, $97.21 \% \pm 1.69 \%$, and $96.91 \% \pm 1.8 \%$ for $\mathrm{Z7}, \mathrm{F} 7$, and $\mathrm{O} 7$, respectively. Even after 60 days of storage at room temperature, no samples had a marked reduction in concentrations of remaining CsA. These results indicate that there were no changes in concentration of remaining CsA during the 60 days. Chemical stability results when stored at $4^{\circ} \mathrm{C}$ also showed a similar tendency.

To summarize, no distinct changes in mean particle size, $\mathrm{pH}$, osmolarity, or drug content were observed during the stability study of MS-CsA. In fact, the MS-CsA that we developed has proven stability and is being marketed in South Korea (Clacier; Alcon Laboratories Inc, Fort Worth, TX, USA). Previously, when we applied to the Korean Food and Drug Administration for drug approval under the name Clacier, we conducted an experiment by adhering to the International Council for Harmonisation guidelines. However, since the focus of this experiment was the formulation, we only assessed its stability within a short period of time. These stability results, which suggest that MSs prepared by the simple method would be stable for long periods of time, indicate that our newly developed micelle formulations meet the requirements for an effective ocular drug-delivery system.

Table 2 Changes in particle size of MS-CsA stored at room temperature and $4^{\circ} \mathrm{C}$

\begin{tabular}{|c|c|c|c|c|c|c|c|c|c|}
\hline & \multicolumn{3}{|c|}{ Ethanol 0 system } & \multicolumn{3}{|c|}{ Ethanol $0.5 \%$ system } & \multicolumn{3}{|c|}{ Ethanol I\% system } \\
\hline & $\mathbf{Z 5}$ & Z6 & $\mathbf{Z 7}$ & F5 & F6 & F7 & 05 & 06 & 07 \\
\hline Day & \multicolumn{9}{|c|}{ Storage at room temperature } \\
\hline 4 & 18.13 & 18.47 & 19.03 & 18.07 & 18.83 & 18.7 & 18.53 & 18.93 & 18.2 \\
\hline 7 & 19.43 & 19.97 & 20.27 & 19.73 & 19.47 & 19.57 & 19.83 & 20.27 & 20.93 \\
\hline 10 & 18.77 & 18.8 & 18.2 & 19.3 & 18.97 & 19.37 & 18.9 & 19.2 & 19.57 \\
\hline 14 & 20.33 & 20.07 & 20.1 & 19.97 & 19.8 & 19.83 & 21.73 & 20 & 19.83 \\
\hline 21 & 20.8 & 20.73 & 20.8 & 21 & 20.37 & 20.13 & 20.6 & 20.8 & 20.07 \\
\hline 30 & 20.8 & 21.03 & 21.17 & 20.63 & 20.53 & 20.37 & 20.4 & 20.1 & 20 \\
\hline \multirow[t]{2}{*}{60} & 18.53 & 19.27 & 19.47 & 19.07 & 19.63 & 19.5 & 19.4 & 19.5 & 18.93 \\
\hline & \multicolumn{9}{|c|}{ Storage at $4^{\circ} \mathrm{C}$} \\
\hline 4 & 18.2 & 18.47 & 18.7 & 18.37 & 18.7 & 19.07 & 17.97 & 17.9 & 18.77 \\
\hline 7 & 18.5 & 18.9 & 18.23 & 18.5 & 19.6 & 19.7 & 20.23 & 19.67 & 19.5 \\
\hline 10 & 18.17 & 19 & 19.33 & 19.4 & 19.47 & 19.53 & 19.7 & 19.9 & 19.83 \\
\hline 14 & 21.73 & 21.87 & 21 & 21.37 & 21.27 & 21.9 & 21.2 & 20.83 & 19.7 \\
\hline 21 & 19.53 & 19.33 & 19.2 & 19.37 & 19.73 & 18.8 & 19.63 & 19.5 & 19.3 \\
\hline 30 & 20.4 & 21.13 & 21.5 & 20.8 & 20.87 & 20.73 & 20.83 & 21.03 & 21.33 \\
\hline 60 & 19.27 & 18.5 & 18.07 & 18.03 & 18.8 & 18.63 & 18.97 & 18.33 & 18.47 \\
\hline
\end{tabular}

Note: Data are expressed as mean $(n=3)$.

Abbreviation: MS-CsA, micelle solution of cyclosporine A. 
Table $3 \mathrm{pH}$ and osmolarity analysis of different micelle formulations

\begin{tabular}{|c|c|c|c|c|c|c|}
\hline \multirow[t]{2}{*}{ Formulation } & \multicolumn{3}{|l|}{$\mathbf{p H}$} & \multicolumn{3}{|c|}{ Osmolarity (mOsm) } \\
\hline & Initial pH & After 60 days & $\begin{array}{l}\text { Increasing } \\
\text { ratio }^{a}\end{array}$ & $\begin{array}{l}\text { Initial } \\
\text { osmolarity }\end{array}$ & After 60 days & $\begin{array}{l}\text { Increasing } \\
\text { ratio }^{\text {b }}\end{array}$ \\
\hline \multicolumn{7}{|c|}{ Stored at room temperature over 60 days } \\
\hline Z5 & $7.4 I \pm 0.0 I$ & $7.39 \pm 0.02$ & 0.997 & $317.4 \pm 7.64$ & $303.7 \pm 4.62$ & 0.957 \\
\hline Z6 & $7.42 \pm 0.02$ & $7.38 \pm 0.06$ & 0.995 & $316.77 \pm 12.86$ & $299.87 \pm 3.26$ & 0.947 \\
\hline Z7 & $7.43 \pm 0.03$ & $7.4 \pm 0.03$ & 0.996 & $311 \pm 10.65$ & $298.57 \pm 3.95$ & 0.96 \\
\hline F5 & $7.43 \pm 0.03$ & $7.4 I \pm 0.03$ & 0.997 & $305.5 \pm 3.51$ & $296.6 \pm 13.67$ & 0.971 \\
\hline F6 & $7.47 \pm 0.02$ & $7.4 I \pm 0.02$ & 0.992 & $298.30 \pm 9.54$ & $304.4 \pm 5.03$ & 1.02 \\
\hline F7 & $7.44 \pm 0.01$ & $7.4 \pm 0.01$ & 0.995 & $299.63 \pm 2.68$ & $296.6 \pm 10.82$ & 0.99 \\
\hline O5 & $7.42 \pm 0.03$ & $7.42 \pm 0.05$ & I & $309.53 \pm 12.11$ & $301.67 \pm 4$ & 0.975 \\
\hline O6 & $7.46 \pm 0.01$ & $7.4 \pm 0.05$ & 0.992 & $311.13 \pm 4.3$ & $297.47 \pm 2.95$ & 0.956 \\
\hline O7 & $7.45 \pm 0.03$ & $7.39 \pm 0.07$ & 0.992 & $306.37 \pm 3.46$ & $300.3 \pm 3.02$ & 0.98 \\
\hline \multicolumn{7}{|c|}{ Stored at $4^{\circ} \mathrm{C}$ over 60 days } \\
\hline Z5 & $7.4 \mathrm{I} \pm 0.0 \mathrm{I}$ & $7.4 \pm 0.01$ & 0.999 & $317.4 \pm 7.64$ & $309.03 \pm 7.8$ & 0.974 \\
\hline Z6 & $7.42 \pm 0.02$ & $7.4 I \pm 0.02$ & 0.999 & $316.77 \pm 12.86$ & $307.17 \pm 7.48$ & 0.97 \\
\hline Z7 & $7.43 \pm 0.03$ & $7.39 \pm 0.09$ & 0.995 & $311 \pm 10.65$ & $304.87 \pm 14.2$ & 0.98 \\
\hline F5 & $7.43 \pm 0.03$ & $7.4 I \pm 0.08$ & 0.997 & $305.5 \pm 3.51$ & $297.7 \pm 7.57$ & 0.974 \\
\hline F6 & $7.47 \pm 0.02$ & $7.37 \pm 0.05$ & 0.987 & $298.3 \pm 9.54$ & $300.13 \pm 2.99$ & 1.006 \\
\hline F7 & $7.44 \pm 0.01$ & $7.4 I \pm 0.04$ & 0.996 & $299.63 \pm 2.68$ & $303.27 \pm 10.05$ & 1.012 \\
\hline O5 & $7.42 \pm 0.03$ & $7.39 \pm 0.06$ & 0.996 & $309.53 \pm 12.11$ & $302 \pm 9.24$ & 0.976 \\
\hline O6 & $7.46 \pm 0.01$ & $7.39 \pm 0.07$ & 0.991 & $311.13 \pm 4.3$ & $298.27 \pm 3.7$ & 0.959 \\
\hline O7 & $7.45 \pm 0.03$ & $7.42 \pm 0.03$ & 0.996 & $306.37 \pm 3.46$ & $300.03 \pm 5.02$ & 0.979 \\
\hline
\end{tabular}

Notes: alncreasing ratio $=\mathrm{pH}$ after 60 days/initial $\mathrm{pH}$; bincreasing ratio $=$ osmolarity after 60 days/initial osmolarity. Data expressed as mean \pm standard deviation $(\mathrm{n}=3$ ). Information about the formulation names is shown in Table I.

\section{Measurements of aqueous tear production}

In this study, we induced dry eyes in rabbits and then confirmed the presence of induced dry eyes in these rabbits. We then saw that CsA eyedrops were able to accelerate the lachrymation, confirming their therapeutic effect. Commonly,
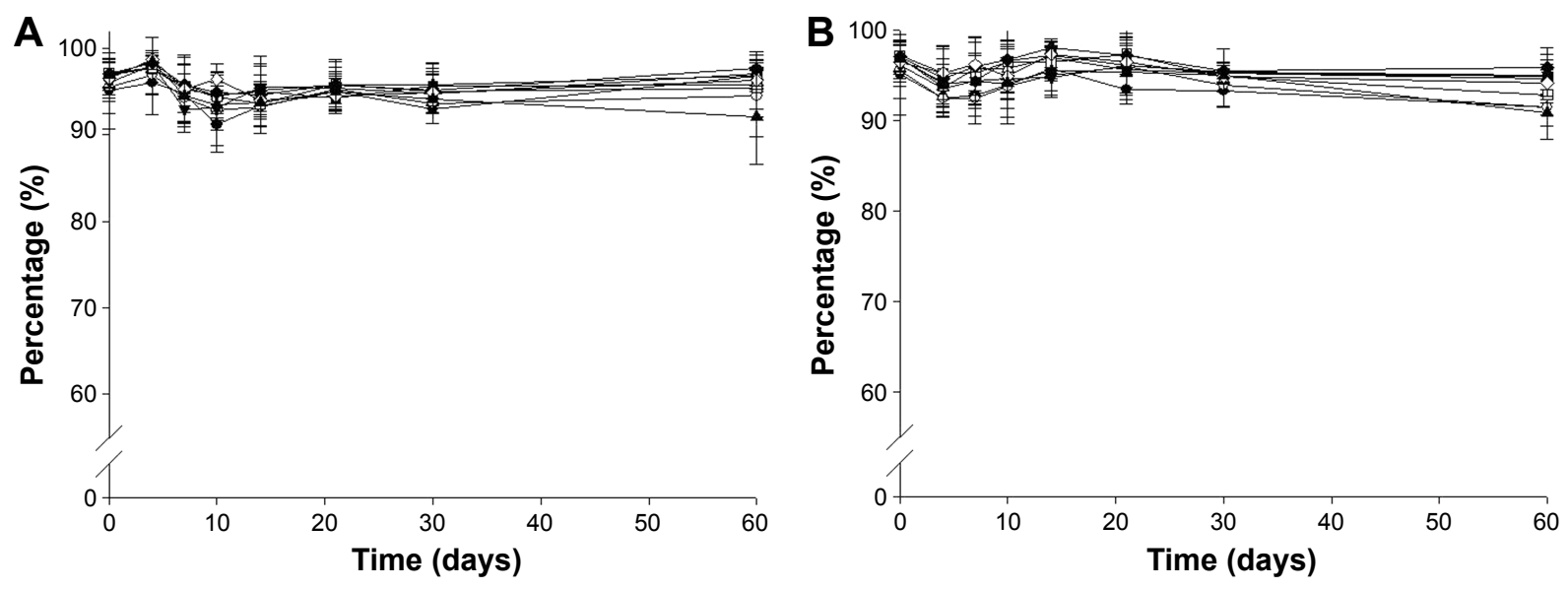

topical administration of AS, which antagonizes all types of muscarinic receptors, rapidly induces typical dry-eye symptoms. ${ }^{22,25}$ Hence, bilateral eyes in rabbits were treated equally with AS solution to induce dry eyes. After induction of dry eyes, one eye was treated with drug formulations (group 1, MS-CsA of O7; group 2, MS-CsA of O4; group 3,

Figure 2 Changes in chemical stability (remaining CsA) of MS-CsA stored at (A) room temperature and (B) $4^{\circ} \mathrm{C}$ at different time intervals over 60 days. Note: Data expressed as mean \pm standard deviation $(n=3)$.

Abbreviation: MS-CsA, micelle solution of cyclosporine A. 

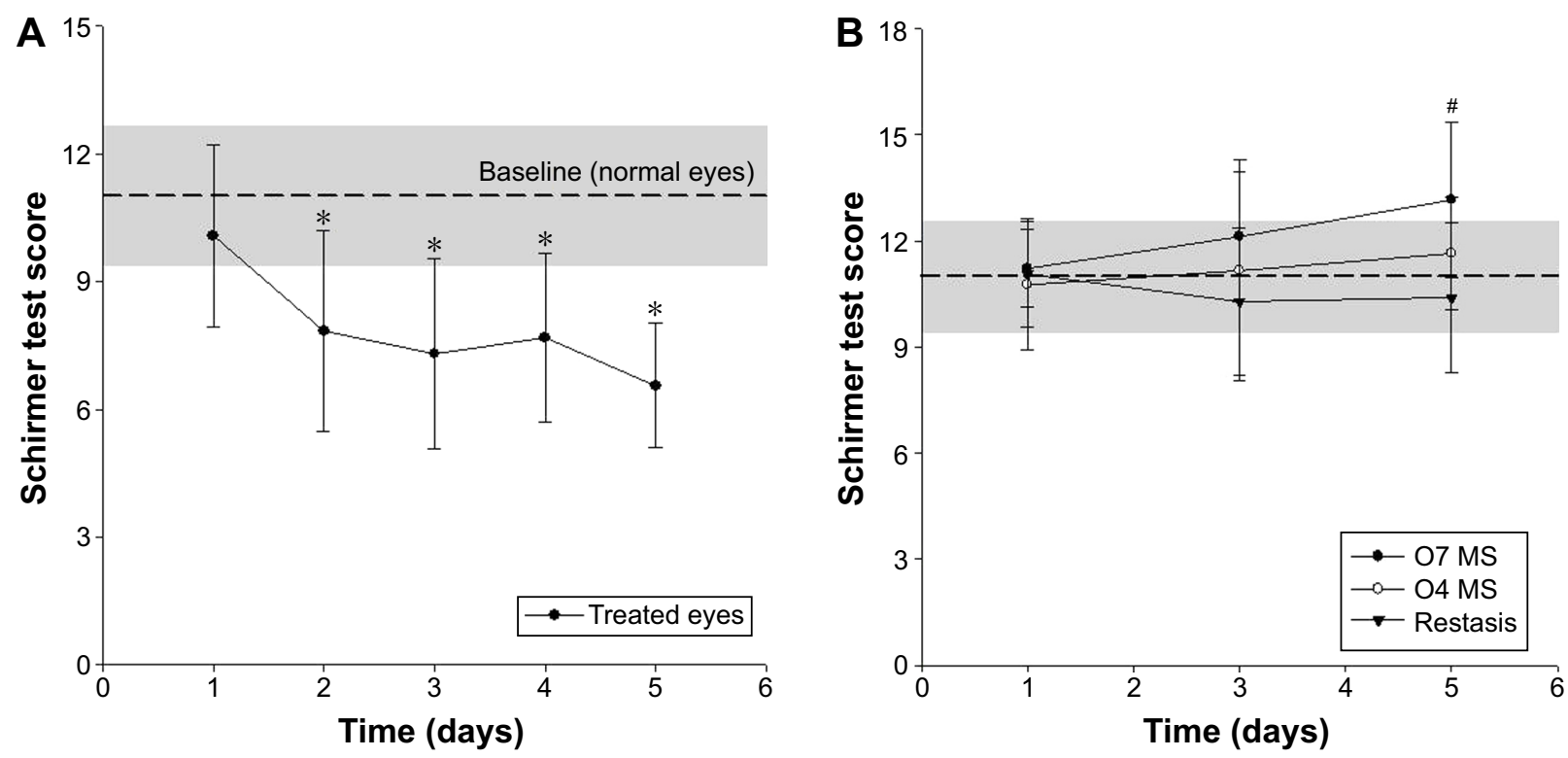

Figure 3 (A) STT scores in the AS-treated group (control) at different time points and (B) according to CsA-formulation treatment in the rabbit dry-eye model. Notes: $* P<0.05$ versus STT score of day I; $\# P<0.05$ (one-way ANOVA between the three groups). Data expressed as mean \pm standard deviation $(n=8)$. Abbreviations: AS, atropine sulfate (I\% solution); STT, Schirmer tear test; CsA, cyclosporine A; MS, micelle solution; ANOVA, analysis of variance.

Restasis), while the other eye was treated with NS. The initial average STT scores of the three groups were defined as the range of normal eyes (baseline). STT scores ranging from 9.38 to 12.65 (average \pm standard deviation) were the range of normal eyes in our study. In Figure 3, the gray box (baseline) indicates the scores corresponding to normal eyes.

The STT scores (reported as millimeters of wet strip 2 minutes after insertion) of the control group are reported in Figure 3A. These show noticeable and significant changes in tear production during 5 days of the in vivo experiment. Decreased tear production was observed on the second day after beginning of treatment. The average STT score was reduced from $10.08 \pm 2.13 \mathrm{~mm}$ (day 1) to $7.85 \pm 2.36$ (day 2) at this time (paired $t$-test, $P=0.035$ ). The STT score of the control group at the end of the experiment was $6.56 \pm 1.47$.

Table 4 summarizes the STT scores and tear-difference values of all three drug-treated groups. On the first day, STT scores between the drug-treated eyes and NS-treated eyes were significantly similar, and scores were around 10 or $11 \mathrm{~mm}$, which are values expected in normal eyes. The tear-difference values of groups $1-3$ were $1.38 \pm 1.79 \mathrm{~mm}$, $0.84 \pm 1.8 \mathrm{~mm}$, and $1.25 \pm 3.55 \mathrm{~mm}$, respectively. Nevertheless, after that, both eyes of the groups treated with MS-CsA in one eye showed a significant difference on day 3 (group 1, $P=0.0007$; group 2, $P=0.0254$ ). After treatment for 5 days, there was significant improvement $(P=0.0032)$ in the STT scores of the Restasis-treated eyes in comparison with untreated eyes. However, the extent of the improvement was much higher in the $\mathrm{O} 7(P=0.0003)$ - and $\mathrm{O} 4(P=0.0003)$ treated groups, which were almost above the initial scores of the respective groups. At the last test point, the teardifference values among group 1, group 2, and group 3 were distinct. The tear-difference values of groups 1-3 were $6.35 \pm 4.49 \mathrm{~mm}, 4.9 \pm 2.96 \mathrm{~mm}$, and $3.6 \pm 3.27 \mathrm{~mm}$, respectively. The tear-difference value of group 1 was approximately 1.76 -fold that of group 3. Compared with the conventional emulsion (Restasis), MS-CsA formulations

Table 4 Schirmer tear test scores with cure

\begin{tabular}{|c|c|c|c|}
\hline & Day I & Day 3 & Day 5 \\
\hline \multicolumn{4}{|l|}{ Group I } \\
\hline $\mathrm{AS}+\mathrm{O7}$ & $11.23 \pm 1.1$ & $12.13 \pm 1.84^{a}$ & $13.16 \pm\left. 2.2\right|^{\mathrm{a}}$ \\
\hline $\mathrm{AS}+\mathrm{NS}$ & $9.85 \pm 1.78$ & $7.78 \pm 2.16$ & $6.81 \pm 3.08$ \\
\hline$P_{1}$ & 0.084 & 0.0007 & 0.0003 \\
\hline Tear-difference value & $1.38 \pm 1.79$ & $4.35 \pm 1.54$ & $6.35 \pm 4.49$ \\
\hline \multicolumn{4}{|l|}{ Group 2} \\
\hline $\mathrm{AS}+\mathrm{O} 4$ & $10.76 \pm 1.86$ & $11.16 \pm 3.12^{\mathrm{b}}$ & $11.65 \pm 1.58^{b}$ \\
\hline $\mathrm{AS}+\mathrm{NS}$ & $9.93 \pm 1.59$ & $7.25 \pm 3.14$ & $6.75 \pm 2.45$ \\
\hline$P_{2}$ & 0.3497 & 0.0254 & 0.0003 \\
\hline Tear-difference value & $0.84 \pm 1.8$ & $3.91 \pm 4.73$ & $4.90 \pm 2.96$ \\
\hline \multicolumn{4}{|l|}{ Group 3} \\
\hline AS + Restasis & $11.06 \pm 1.5$ & $10.29 \pm 2.07$ & $10.4 \pm 2.1 I^{c}$ \\
\hline $\mathrm{AS}+\mathrm{NS}$ & $9.8 \mathrm{I} \pm 2.74$ & $7.58 \pm 3.44$ & $6.8 \pm 1.94$ \\
\hline$P_{3}$ & 0.2763 & 0.0767 & 0.0032 \\
\hline Tear-difference value & $1.25 \pm 3.55$ & $2.7 I \pm 3.12$ & $3.6 \pm 3.27$ \\
\hline
\end{tabular}

Notes: ${ }^{a} \mathrm{P}<0.05$ versus AS + NS in group I on the same day; ${ }^{b} \mathrm{P}<0.05$ versus AS + NS in group 2 on the same day; ${ }^{c} P<0.05$ versus AS + NS in group 3 on the same day. Data expressed as mean \pm standard deviation $(n=8)$. All solutions administered at $50 \mu \mathrm{L}$.

Abbreviations: AS, atropine sulfate (I\% solution); NS, normal saline. 
showed improvement demonstrated by the tear-difference values between day 1 and day 5 .

STT scores relevant to treatment with the drug formulations under testing are illustrated in Figure 3B. On the first day, the STT scores of all groups were significantly similar (ANOVA, $P=0.8276$ ) and belonged to the normal-eye range. On the fifth day, one-way ANOVA $(P=0.0365)$ showed that there were statistically significant differences among the formulations posttreatment, and the therapeutic effectiveness of the formulations (based on the STT scores) was possible to predict. Interestingly, it was observed that only O7 produced greater scores with respect to normal eyes at the last experimental time, while the other two formulations produced scores within normal ranges during all experimental times. The enhanced effect of micelle formulations might be due to the increased permeability in relation to the amount of Cremophor EL. These results suggest that the micelle formulations in this study have the potential to reduce the amount of active pharmaceutical ingredients.

\section{Goblet-cell density}

Lachrymal glands, conjunctivas, and corneas are affected in dry-eye disease. Therefore, the ocular surface is the ideal target for topical administration of CsA in order to suppress ocular inflammation without significant systemic CsA exposure. ${ }^{38}$ Goblet cells are located in the surface of conjunctiva-secreted mucin, and once it is secreted, it possesses the ability to hydrate and gel the conjunctiva. ${ }^{39}$ This biological film produces a protective covering over the ocular surface ${ }^{40,41}$ to protect it from a variety of pathogens, chemicals, and environmental toxins. ${ }^{39}$ Conjunctival goblet cells are the main source of ocular surface mucoproteins that lubricate and protect the ocular surface. ${ }^{42}$ It is likely that the poorly lubricated ocular surface resulting from reduced mucin production makes the tear film unstable. ${ }^{43}$ Therefore, goblet-cell density (adequate numbers of goblet cells) is a critical parameter that reflects the overall health of the ocular surface. ${ }^{43-46}$ CsA emulsion (Restasis), but not artificial tears, increases goblet-cell density in the bulbar conjunctiva in patients with dry eyes. ${ }^{24,45}$

To evaluate the effects of sequential treatment with AS and drug formulations on conjunctival goblet-cell density in the rabbit, samples of conjunctiva sacs were stained with H\&E solution. Table 5 shows the number of average conjunctival goblet cells of groups 1 and 3 . The number of goblet

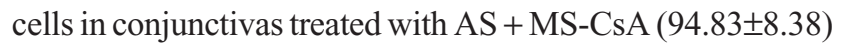
or AS + Restasis $(65.17 \pm 11.51)$ was significantly higher than the number of goblet cells treated with AS + NS (group 1,
Table 5 Average conjunctival goblet-cell density

\begin{tabular}{lll}
\hline & Goblet-cell density & Difference \\
\hline Group I & & \\
AS + O7 & $94.83 \pm 8.38^{\mathrm{a}, \mathrm{b}}$ & $51.17 \pm 6.43$ \\
AS + NS & $43.67 \pm 4.76$ & \\
Group 3 & & \\
AS + Restasis & $65.17 \pm 11.5 \mathrm{I}^{\mathrm{c}}$ & $24.67 \pm 9.33$ \\
AS + NS & $40.5 \pm 7.0 \mathrm{I}$ & \\
\hline
\end{tabular}

Notes: $\mathrm{P}<0.05$ versus AS + NS in group I; $\mathrm{b}<0.05$ versus AS + Restasis in group 3; $c P<0.05$ versus $A S+N S$ in group 3. Data expressed as mean \pm standard deviation ( $\mathrm{n}=6$, three separate tissues by two independent masked observers).

Abbreviations: AS, atropine sulfate (I\% solution); NS, normal saline.

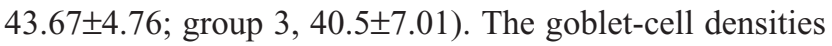
of group 1 (treated with O7) and group 3 (treated with Restasis) showed a significant difference $(P<0.05)$. As a result, MS-CsA formulations have the potential to become a useful tool for curing dry eyes, and could be employed as an ideal alternative to Restasis for the treatment of dry-eye syndrome.

\section{Conjunctival epithelial morphology}

As shown in Figure 4, goblet cells were stained purple and located in the superficial epithelium. Treatment with AS decreased goblet-cell density, such as dry-eye disease. Also, these results were consistent with STT results, as previously mentioned.

Conjunctivas of rabbits after 5 days of treatment with AS and NS are shown in Figure 4, C (group 1) and E (group 3), respectively. These epithelia were thinned with the loss of goblet cells. However, conjunctivas of rabbits after 5 days of treatment with AS and drug formulations recovered epithelia, and there was a relative increase in the number of goblet cells. Conjunctivas treated with MS-CsA formulation (Figure 4D) or commercial CsA emulsion (Figure 4F) were composed of sufficient layers of epithelial cells and contained numerous goblet cells.

\section{Conclusion}

CsA eyedrops are widely known as a therapeutic agent for dry-eye syndrome in the pharmaceutical industry. However, alternative preparations of ophthalmic medications for this disorder, except for Restasis, are almost nonexistent, due to stability and patient-compliance issues. To develop transparent MS-CsA capable of enhancing patient compliance and effectively treating patient with dry-eye syndrome, MS-CsA formulations composed of nonionic surfactant Cremophor EL, ethanol, and phosphate buffer were established. These studies revealed that the MS-CsA 

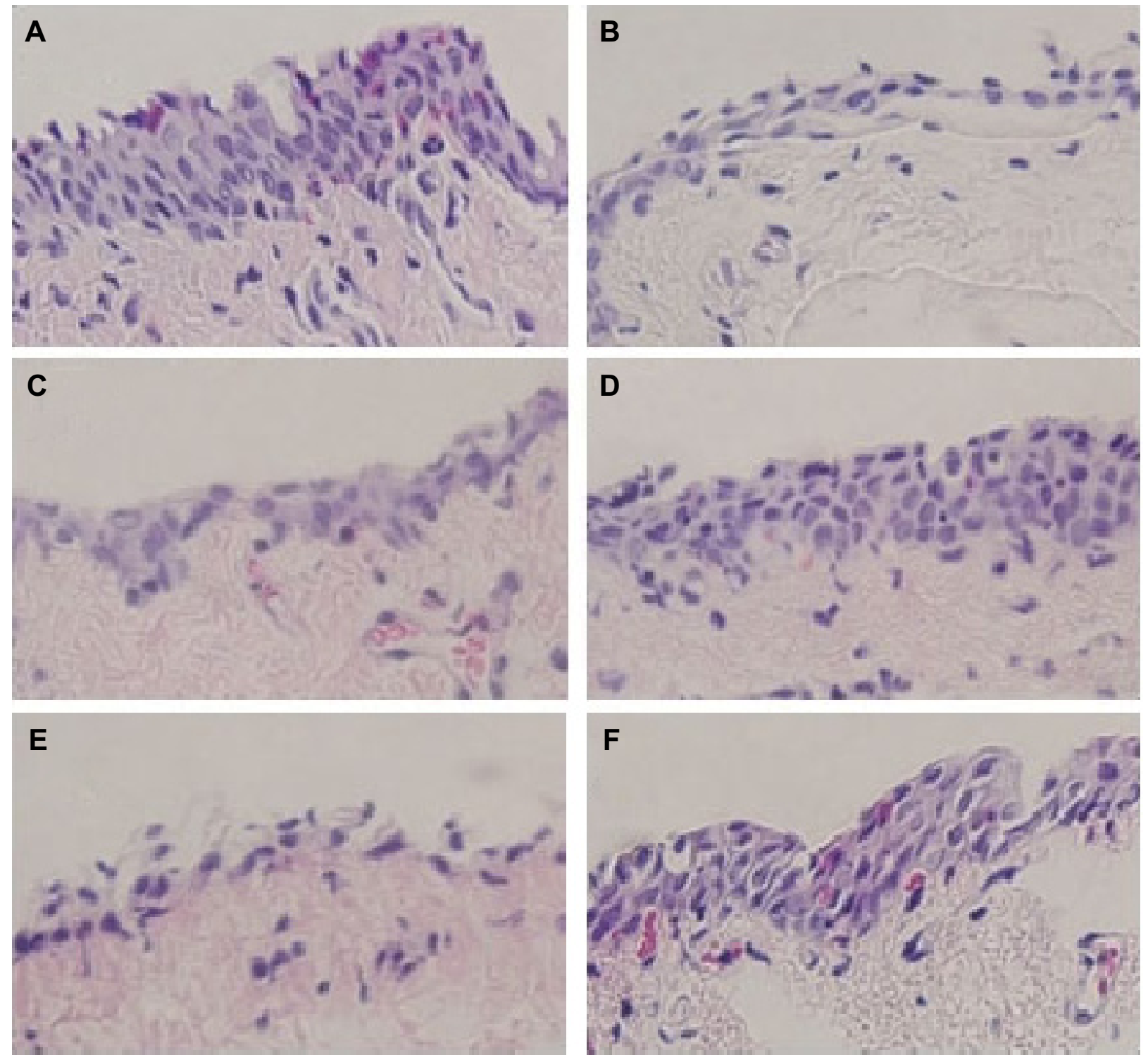

Figure 4 Images of histologic sections from the rabbit conjunctiva stained with H\&E.

Notes: (A) Administration of normal saline only; (B) administration of $1 \%$ atropine sulfate solution only; (C) AS + NS administration in group I; (D) AS + O7 administration in group I; (E) AS + NS administration in group 3; (F) AS + Restasis administration in group 3. Goblet cells are stained purple and located in the superficial epithelium. Epithelia in (B), (C), and (E) were thinned with loss of goblet cells. Goblet-cell morphology in (D) was relatively well formed. Magnification 40×. All solutions administered at $50 \mu \mathrm{L}$.

Abbreviations: AS, atropine sulfate (I\% solution); H\&E, hematoxylin and eosin; NS, normal saline.

manufacturing method provided a simpler and more efficient way to prepare eyedrops that were characteristically much smaller and with more homogeneous size distribution than Restasis. MS-CsA can be stored for 60 days without any chemical or physical changes, such as $\mathrm{pH}$, osmolarity, particle size, and content. The newly developed MS-CsA showed significant therapeutic effect, as demonstrated by histological evidence of higher goblet-cell density and clinical evidence of improved tear production in dry eyes. Our results strongly implicate that MS-CsA could be an ideal alternative therapy for dry eyes. Furthermore, the MS-CsA delivery system, with its shorter processing time and possible large-scale production, is an advantageous technique.

\section{Acknowledgments}

This work was supported by the Mid-career Researcher Program through a National Research Foundation grant funded by the Ministry of Science, ICT, and Future Planning, South Korea (NRF-2014R1A2A2A01005059). The authors were also assisted by Byung Joon Park, who helped with the English revisions and contributed ideas to the "Results and discussion" section. 


\section{Disclosure}

The authors report no conflicts of interest in this work.

\section{References}

1. Lallemand F, Felt-Baeyens O, Besseghir K, Behar-Cohen F, Gurny R. Cyclosporine A delivery to the eye: a pharmaceutical challenge. Eur $J$ Pharm Biopharm. 2003;56(3):307-318.

2. Kashani S, Mearza AA. Uses and safety profile of ciclosporin in ophthalmology. Expert Opin Drug Saf. 2008;7(1):79-89.

3. Karn PR, Cho W, Park HJ, Park JS, Hwang SJ. Characterization and stability studies of a novel liposomal cyclosporin A prepared using the supercritical fluid method: comparison with the modified conventional Bangham method. Int J Nanomedicine. 2013;8:365-377.

4. Rodriguez-Aller M, Kaufmann B, Guillarme D, et al. In vivo characterisation of a novel water-soluble cyclosporine A prodrug for the treatment of dry eye disease. Eur J Pharm Biopharm. 2012;80(3): 544-552.

5. Noble S, Markham A. Cyclosporin: a review of the pharmacokinetic properties, clinical efficacy and tolerability of a microemulsion-based formulation (Neoral). Drugs. 1995;50(5):924-941.

6. Mondon K, Zeisser-Labouèbe M, Gurny R, Möller M. Novel cyclosporin A formulations using MPEG-hexyl-substituted polylactide micelles: a suitability study. Eur J Pharm Biopharm. 2011;77(1):56-65.

7. Di Tommaso C, Bourges JL, Valamanesh F, et al. Novel micelle carriers for cyclosporin A topical ocular delivery: in vivo cornea penetration, ocular distribution and efficacy studies. Eur J Pharm Biopharm. 2012; 81(2):257-264.

8. Strickley RG. Solubilizing excipients in oral and injectable formulations. Pharm Res. 2004;21(2):201-230.

9. Javadi MA, Feizi S. Dry eye syndrome. J Ophthalmic Vis Res. 2011;6(3): 192-198.

10. Pflugfelder SC. Antiinflammatory therapy for dry eye. Am J Ophthalmol. 2004;137(2):337-342.

11. Schaumberg DA, Sullivan DA, Buring JE, Dana MR. Prevalence of dry eye syndrome among US women. Am J Ophthalmol. 2003;136(2): 318-326.

12. Karn PR, Kim HD, Kang H, Sun BK, Jin SE, Hwang SJ. Supercritical fluid-mediated liposomes containing cyclosporin A for the treatment of dry eye syndrome in a rabbit model: comparative study with the conventional cyclosporin A emulsion. Int J Nanomedicine. 2014;9: 3791-3800

13. Gupta C, Chauhan A. Ophthalmic delivery of cyclosporine A by punctal plugs. J Control Release. 2011;150(1):70-76.

14. Aliabadi HM, Brocks DR, Lavasanifar A. Polymeric micelles for the solubilization and delivery of cyclosporine A: pharmacokinetics and biodistribution. Biomaterials. 2005;26(35):7251-7259.

15. Kuwano M, Ibuki H, Morikawa N, Ota A, Kawashima Y. Cyclosporine A formulation affects its ocular distribution in rabbits. Pharm Res. 2002; 19(1):108-111.

16. Luschmann C, Tessmar J, Schoeberl S, Strauss O, Luschmann K, Goepferich A. Self-assembling colloidal system for the ocular administration of cyclosporine A. Cornea. 2014;33(1):77-81.

17. Cholkar K, Gilger BC, Mitra AK. Topical, aqueous, clear cyclosporine formulation design for anterior and posterior ocular delivery. Transl Vis Sci Technol. 2015;4(3):1.

18. Jiao J. Polyoxyethylated nonionic surfactants and their applications in topical ocular drug delivery. Adv Drug Deliv Rev. 2008;60(15): $1663-1673$.

19. Roggeband R, York M, Pericoi M, Braun W. Eye irritation responses in rabbit and man after single applications of equal volumes of undiluted model liquid detergent products. Food Chem Toxicol. 2000;38(8): 727-734.

20. Ammar HO, Salama HA, Ghorab M, Mahmoud AA. Nanoemulsion as a potential ophthalmic delivery system for dorzolamide hydrochloride. AAPS PharmSciTech. 2009;10(3):808-819.
21. van der Bijl P, van Eyk AD, Meyer D. Effects of three penetration enhancers on transcorneal permeation of cyclosporine. Cornea. 2001; 20(5):505-508

22. Burgalassi S, Panichi L, Chetoni P, Saettone MF, Boldrini E. Development of a simple dry eye model in the albino rabbit and evaluation of some tear substitutes. Ophthalmic Res. 1999;31(3):229-235.

23. Gin J, Wong VG, inventors; Visionex, assignee. Schirmer tear test. United States patent US 5006310. 1991 Apr 9.

24. Kunert KS, Tisdale AS, Gipson IK. Goblet cell numbers and epithelial proliferation in the conjunctiva of patients with dry eye syndrome treated with cyclosporine. Arch Ophthalmol. 2002;120(3):330-337.

25. Dursun D, Wang M, Monroy D, et al. A mouse model of keratoconjunctivitis sicca. Invest Ophthalmol Vis Sci. 2002;43(3):632-638.

26. Stevenson D, Tauber J, Reis BL. Efficacy and safety of cyclosporin A ophthalmic emulsion in the treatment of moderate-to-severe dry eye disease: a dose-ranging, randomized trial. Ophthalmology. 2000; 107(5):967-974.

27. van der Bijl P, Engelbrecht AH, Van Eyk AD, Meyer D. Comparative permeability of human and rabbit corneas to cyclosporin and tritiated water. J Ocul Pharmacol Ther. 2002;18(5):419-427.

28. Das S, Mondal S, Ghosh S. Physicochemical studies on the micellization of cationic, anionic, and nonionic surfactants in water-polar organic solvent mixtures. J Chem Eng Data. 2013;58(9):2586-2595.

29. van Tellingen O, Huizing MT, Panday VR, Schellens JH, Nooijen WJ, Beijnen JH. Cremophor EL causes (pseudo-) non-linear pharmacokinetics of paclitaxel in patients. Br J Cancer. 1999;81(2):330-335.

30. Torchilin VP. Micellar nanocarriers: pharmaceutical perspectives. Pharm Res. 2007;24(1):1-16.

31. Bonanno JA, Polse KA. Measurement of in vivo human corneal stromal pH: open and closed eyes. Invest Ophthalmol Vis Sci. 1987;28(3): $522-530$.

32. Coles WH, Jaros PA. Dynamics of ocular surface pH. Br J Ophthalmol. 1984;68(8):549-552.

33. Missel PJ, Lang JC, Rodeheaver DP, et al. Design and evaluation of ophthalmic pharmaceutical products. In: Florence AT, Siepmann J, editors. Modern Pharmaceutics. 5th ed. Boca Raton (FL): CRC Press; 2009:101-189.

34. Suzuki M, Massingale ML, Ye F, et al. Tear osmolarity as a biomarker for dry eye disease severity. Invest Ophthalmol Vis Sci. 2010;51(9): 4557-4561.

35. Beauchesne PR, Chung NS, Wasan KM. Cyclosporine A: a review of current oral and intravenous delivery systems. Drug Dev Ind Pharm. 2007;33(3):211-220.

36. el Tayar N, Mark AE, Vallat P, Brunne RM, Testa B, van Gunsteren WF. Solvent-dependent conformation and hydrogen-bonding capacity of cyclosporin A: evidence from partition coefficients and molecular dynamics simulations. J Med Chem. 1993;36(24):3757-3764.

37. Mithani SD, Bakatselou V, TenHoor CN, Dressman JB. Estimation of the increase in solubility of drugs as a function of bile salt concentration. Pharm Res. 1996;13(1):163-167.

38. Acheampong AA, Shackleton M, Tang-Liu DD, Ding S, Stern ME, Decker R. Distribution of cyclosporin A in ocular tissues after topical administration to albino rabbits and beagle dogs. Curr Eye Res. 1999; 18(2):91-103

39. Shatos MA, Rios JD, Horikawa Y, et al. Isolation and characterization of cultured human conjunctival goblet cells. Invest Ophthalmol Vis Sci. 2003;44(6):2477-2486.

40. Chao CC, Butala SM, Herp A. Studies on the isolation and composition of human ocular mucin. Exp Eye Res. 1988;47(2):185-196.

41. Holly FJ, Lemp MA. Wettability and wetting of corneal epithelium. Exp Eye Res. 1971;11(2):239-250.

42. Watanabe H. Significance of mucin on the ocular surface. Cornea. 2002; 21(2 Suppl 1):S17-S22.

43. Xiong C, Chen D, Liu J, et al. A rabbit dry eye model induced by topical medication of a preservative benzalkonium chloride. Invest Ophthalmol Vis Sci. 2008;49(5):1850-1856. 
44. Barabino S, Shen L, Chen L, Rashid S, Rolando M, Dana MR. The controlled-environment chamber: a new mouse model of dry eye. Invest Ophthalmol Vis Sci. 2005;46(8):2766-2771.

45. Pflugfelder SC, De Paiva CS, Villarreal AL, Stern ME. Effects of sequential artificial tear and cyclosporine emulsion therapy on conjunctival goblet cell density and transforming growth factor- $\beta 2$ production. Cornea. 2008;27(1):64-69.
46. Kinoshita S, Kiorpes TC, Friend J, Thoft RA. Goblet cell density in ocular surface disease: a better indicator than tear mucin. Arch Ophthalmol. 1983;101(8):1284-1287.

\section{Publish your work in this journal}

The International Journal of Nanomedicine is an international, peerreviewed journal focusing on the application of nanotechnology in diagnostics, therapeutics, and drug delivery systems throughout the biomedical field. This journal is indexed on PubMed Central, MedLine, CAS, SciSearch $\AA$, Current Contents ${ } /$ Clinical Medicine,
Journal Citation Reports/Science Edition, EMBase, Scopus and the Elsevier Bibliographic databases. The manuscript management system is completely online and includes a very quick and fair peer-review system, which is all easy to use. Visit http://www.dovepress.com/ testimonials.php to read real quotes from published authors.

Submit your manuscript here: http://www.dovepress.com/international-journal-of-nanomedicine-journal 